\title{
Kampf gegen Armut
}

\section{Die Europäische Plattform gegen Armut und soziale Ausgrenzung}

\section{BERND SCHULTE}

Dr. Bernd Schulte war bis zu seiner Pensionierung im letzten Jahr am Max-Planck-Institut für Sozialrecht und Sozialpolitik tätig.

E-Mail dr.bernd.schulte@t-online.de

\author{
Die Europäische Kommission hat den Kampf gegen \\ die Armut in den Mittelpunkt ihrer Strategie \\ "Europa 2020" gerückt. Bis 2020 soll mindestens \\ 20 Millionen Menschen ein Weg aus Armut und \\ sozialer Ausgrenzung eröffnet werden.
}

Die »Europäische Plattform gegen Armut und soziale Ausgrenzung « ist eine sozialpolitische Initiative der Europäischen Union. (1) Dieses Dokument ist Teil der Europäischen Strategie zur Bekämpfung von Armut und sozialer Ausgrenzung, die nicht zufällig bereits am Beginn der Herausbildung einer originären Europäischen Sozialpolitik in der zweiten Hälfte der 1970er Jahre gestanden hat. Aus diesem Grunde erscheint es angebracht, zu Beginn einen kurzen Blick zurück auf die Entwicklung der Europäischen Politik zur Bekämpfung von Armut und sozialer Ausgrenzung zu werfen.

Im EWG-Vertrag von 1957 war die Sozialpolitik lediglich ein Annex der Wirtschaftspolitik, die seinerzeit unter dem Vorzeichen der Schaffung eines Gemeinsamen Marktes im Mittelpunkt des Europäischen Integrationsprozesses stand. Die Vorschriften über die soziale Sicherheit der Wanderarbeitnehmer (2) sowie die - partielle - Gleichbehandlung von Männern und Frauen unter dem Vorzeichen der Entgeltgleichheit (»gleicher Lohn für gleich[wertig]e Arbeit«) (3) waren zur Förderung der Mobilität des »Faktors Arbeit « und der Freizügigkeit der Arbeitnehmer sowie zur Gewährleistung gleicher Wettbewerbsbedingungen für Unternehmen, die in höherem Maße als andere Frauen beschäftigten, getroffene sozialpolitische Regelungen. Die eigentlichen Vorschriften über die Sozialpolitik (4) -Europäische Sozialpolitik im engeren Sinne - beschränkten sich im Übrigen auf die Unterstützung der Sozialpolitiken der Mitgliedstaaten der damaligen Sechsergemeinschaft und später dann auch der erweiterten Europäischen (Wirtschafts-) Gemeinschaft.

War der Stellenwert des Titels »Sozialpolitik « und damit zugleich des »Sozialen« überhaupt im Europäischen Gemeinschaftsrecht mithin ursprünglich sehr viel schwächer ausgestaltet als andere Aufgabenbereiche, so erfuhr die soziale Dimension auf Gemeinschaftsebene eine erste Aufwertung Anfang der 1970er Jahre. Auf ihrem Gipfel in Paris im Oktober 1972 erteilten die Staats- und Regierungschefs der Mitgliedstaaten der Europäischen Kommission den Auftrag, ein Sozialpolitisches Aktionsprogramm auszuarbeiten, das nach seiner Erstellung vom Rat der Europäischen Gemeinschaften am 21.1.1974 verabschiedet worden ist (5), in einer Zeit, die durch Abschwächung des Wirtschaftswachstums und der Konjunktur ganz allgemein infolge des »Ölschocks« von 1973 gekennzeichnet war.

Dieses Aktionsprogramm enthielt eine ausdrückliche Absage an eine gemeinschaftliche Sozialpolitik im engeren Sinne., betonte jedoch zugleich, dass es eine Reihe sozialpolitischer Probleme gebe, die alle Mitgliedstaaten gleichermaßen beträfen und die deshalb auch am besten auf Gemeinschaftsebene angegangen würden. $\mathrm{Zu}$ diesen Gemeinschaftsaufgaben gehörte auch ein Programm zur Bekämpfung der Armut. (6) Diskutiert wurde seinerzeit bereits über 
eine finanzielle Beteiligung der Europäischen Gemeinschaft an den nationalen Mindesteinkommensystemen, um eine Annäherung der entsprechenden Leistungssysteme der Mitgliedstaaten zu erleichtern, u. a. um diesbezüglich noch über keine entsprechenden Leistungssysteme verfügende Staaten - seinerzeit Griechenland, Portugal und Spanien - dabei zu helfen, wirksame Basissicherungssysteme aufzubauen und um damit zugleich der Vision der Europäischen Gemeinschaft als Sozialgemeinschaft konkretere Züge zu verleihen. (7)

Auf nationaler Ebene und insbesondere auch in Deutschland hat diese Initiative der Europäischen Gemeinschaft zur Bekämpfung der Armut zunächst einmal zur »Wiederentdeckung der Armut" geführt. (8)

Die - rechtlich unverbindliche - Gemeinschaftscharta der sozialen Grundrechte der Arbeitnehmer aus dem Jahre 1989 (9) war die politische Reaktion auf die erste "Revision « des Europäischen Primärrechts in Gestalt des EWG-Vertrages durch die Einheitliche Europäische Akte von 1987, die die Weichen gestellt hat für das Europäische »Binnenmarktprojekt «, dabei jedoch jegliche Stärkung der sozialen Dimension vermissen ließ (was vor allem seinerzeit von den »linken « Parteien, den Gewerkschaften, den Kirchen, den Wohlfahrtsverbänden und der Zivilgesellschaft überhaupt moniert worden ist).

Die Gemeinschaftscharta war zudem noch dem traditionellen Konzept einer »ökonomistischen « Europäischen Sozialpolitik verschrieben, wie sich exemplarisch an Art. 1 der Charta ablesen lässt, welche das Recht der Arbeitnehmer auf Freizügigkeit zum Gegenstand hatte (während die Charta der Grundrechte der Europäischen Union aus dem Jahre 2000 - rechtsverbindlich seit dem 1. Dezember 2009 - wie das deutsche Grundgesetz und wortgleich die Unantastbarkeit der Menschenwürde in ihrem ersten Artikel niedergelegt und damit an den Anfang des Europäischen Grundrechtekatalogs gesetzt hat.

Als Reaktion auf die politische Diskussion um die Umsetzung der Gemeinschaftscharta der sozialen Grundrechte »der Arbeitnehmer« (!) hat der Rat der Europäischen Gemeinschaften im Jahre 1992 zwei Empfehlungen verabschiedet, die zum einen die Vereinbarung ge- meinsamer Kriterien für ausreichende Zuwendungen und Leistungen im Rahmen der Systeme der sozialen Sicherheit (10) im Sinne der Gewährleistung eines sozialen Existenzminimums und zum anderen die Annäherung der Ziele und der Politiken im Bereich des sozialen Schutzes (11) zum Gegenstand hatten.

In der erstgenannten sogenannten "Mindesteinkommens-Empfehlung ", die auch bereits von der »Bekämpfung der sozialen Ausgrenzung « spricht, wird - dem Rechtscharakter des Instruments der Empfehlung entsprechend rechtlich unverbindlich - vorgeschlagen, dass jeder Mensch einen grundlegenden Anspruch auf ausreichende Zuwendungen und Leistungen haben solle, um ein menschenwürdiges Leben führen zu können. Darüber hinaus sollten die Systeme der sozialen Sicherung bestimmten Grundsätzen und Grundorientierungen angepasst werden: Rechtsanspruch; Grundsatz der Bedarfsdeckung; Bedarfsprüfung; Nachrang; Familiengerechtigkeit der Hilfe; Einbeziehung in die primäre soziale Sicherung; Integrationsprinzip; Verknüpfung mit sonstigen Hilfen; Bürgernähe der Verwaltung; Verfahren und Rechtschutz. (12)

Die Mindesteinkommens-Empfehlung von 1992 ist nach wie vor Maßstab der von der Europäischen Union propagierten Mindesteinkommenspolitik, die bis zum heutigen Tage in der politischen Strategie der sogenannten Offenen Methode der Koordinierung (OMK) weiter verfolgt wird. Die Methode wird seit dem Europäischen Rat Lissabon 2000 auch im Bereich des Sozialschutzes als politischer Prozess durchgeführt, weil die klassische »Gemeinschaftsmethode« der Rechtsetzung angesichts der Kompetenzverteilung zwischen Union und Mitgliedstaaten hier nicht zu greifen vermag.

Auch eine Reihe anderer Europäischer Rechtsinstrumente tragen dazu bei, dass auch die Mindestsicherung in stärkerem Maße »europäisiert" worden ist. $\mathrm{Zu}$ nennen sind in diesem $\mathrm{Zu}$ sammenhang die Verordnungen (EG) Nr. 883/2004 und 987/2009 über die soziale Sicherheit als Rechtsgrundlagen der Koordinierung der Systeme der sozialen Sicherheit der Mitgliedstaaten. Zwar sind diese Verordnungen nicht anwendbar auf die »soziale und medizinische Fürsorge« (13) und damit auf die soziale Grundsicherung in Gestalt der Sozialhilfe, doch hat der Europäische Gerichtshof in seiner Judikatur diesen Ausnahmetatbestand eben so ausgelegt, dass sozialhilfeähnliche Regelungen - etwa Leistungen der sozialen Grundsicherung im Alter oder bei Erwerbsunfähigkeit i. S. des SGB XII dem Europäischen Koordinierungsrecht unterliegen, wenn auch ihrem besonderen Charakter, nämlich der Bindung an das wirtschaftliche und soziale Umfeld, dadurch Rechnung getragen wird, dass der »Export « ausgeschlossen werden kann.

Wanderarbeitnehmer werden im Übrigen im Hinblick auf »soziale und steuerliche Vergünstigungen «, zu denen auch die Sozialhilfe gehört, einheimischen Arbeitnehmern gleichgestellt mit der Folge, dass sie gegebenenfalls auch Leistungen der Mindestsicherung beanspruchen können. (14)

Auch die Unionsbürgerschaft (Art. 18 ff. AEUV), die den Bürgern der Mitgliedstaaten der Union einen zusätzlichen »Europäischen « Rechtsstatus verleiht und ihnen insbesondere das Recht auf Freizügigkeit gewährt, vermag Rechtsgrundlage für Ansprüche auf Sozialleistungen einschließlich Mindestsicherungsleistungen sein, die weder über den Arbeitnehmerstatus im vorstehend bezeichneten Sinne noch über den Status als Versicherter im Sinne des europäischen Koordinierungsrechts (15) vermittelt werden. (16)

Die genannten Regelungen sind Ausdruck der zunehmenden Stärkung der sozialen Dimension auf europäischer Ebene. Gemäß Art. 34 Der Europäischen Grundrechte-Charta (EUGRCh) (Recht auf soziale Sicherheit und soziale Unterstützung) anerkennt und achtet die Union, »um die soziale Ausgrenzung und die Armut zu bekämpfen, (...) das Recht auf eine soziale Unterstützung und eine Unterstützung für die Wohnung, die allen, die nicht über ausreichende Mittel verfügen, ein menschenwürdiges Dasein sicherstellen sollen, nach Maßgabe des Unionsrechts und der einzelstaatlichen Rechtsvorschriften und Gepflogenheiten.«(Abs. 3).

\section{Ein europäischer Rahmen für den sozialen und territorialen Zusammenhalt}

Die vorstehenden Ausführungen dürften gezeigt haben, dass der Kampf gegen 
Armut und soziale Ausgrenzung neben der allgemeinen Förderung sozialer Gerechtigkeit und der Respektierung der Menschen- und Grundrechte seit langem zu den Zielsetzungen der Europäischen Union gehört. (17)

Die Europäische Kommission hat den Kampf gegen die Armut auch in den Mittelpunkt ihrer Strategie »Europa 2020 « gerückt, nachdem sich die Staats- und Regierungschefs auf das Ziel geeinigt haben, in der Europäischen Union bis 2020 mindestens 20 Millionen Menschen einen Weg aus der Armut und der sozialen Ausgrenzung $\mathrm{zu}$ eröffnen. Dies geschieht vor dem Hintergrund, dass im Jahre 2008 über 80 Millionen Menschen, also rund 16,5 Prozent der gesamten EU-Bevölkerung unter der Armutsgrenze lebten.

Wie in den 1970er Jahren sollen Wirtschaftswachstum und ein Mehr und Besser an Arbeitsplätzen (obwohl dieses »Besser« m.E. vernachlässigt wird) ein - wohl letztlich »der« Schlüssel im Kampf gegen Armut und soziale Ausgrenzung sein. Konkretisiert wird diese Zielsetzung durch ein Beschäftigungsziel - Mindesterwerbsquote von 75 Prozent für Frauen und Männer im Alter zwischen 20 und 64 - und ein konkretes Bildungsziel - Senkung der Schulabbrecherquote von derzeit 15 auf unter 10 Prozent. (18) Die Plattform greift damit Initiativen auf, die bereits in der Vergangenheit von der Europäischen Kommission vorgeschlagen worden sind. Dies gilt beispielsweise für die aktive Eingliederung der arbeitsmarktfernsten Personen (19) oder die Appelle zur Gewährleistung von Angemessenheit und Nachhaltigkeit der Alterssicherungssysteme als Beitrag zur Vorbeugung und zur Beseitigung von Altersarmut.

$\mathrm{Zu}$ den von der Kommission jetzt in Aussicht genommenen Maßnahmen gehören u. a.

- ein Weißbuch zu Pensionen und Renten, in dem es um die Nachhaltigkeit und Angemessenheit geht

- eine Europäische Innovationspartnerschaft zum aktiven und gesunden Altern im Europäischen Jahr des aktiven Alterns (2012)

- ein europäischer freiwilliger Qualitätsrahmen für soziale Dienstleistungen, u. a. in den Bereichen Langzeitpflege und Wohnungslosigkeit
- die Evaluierung der Effizienz und Effektivität von Gesundheitsausgaben

- eine Mitteilung und einen Vorschlag für eine Empfehlung des Rates zu Strategien gegen Schulabbruch

- eine Empfehlung zur Kinderarmut mit gemeinsamen Grundsätzen und mit Instrumenten für die begleitende Kontrolle

- eine neue europäische Agenda zur Integration von Drittstaatsangehörigen

- ein Rahmen für nationale Strategien zur Eingliederung der Roma

- die Förderung der wirtschaftlichen Unabhängigkeit von Frauen im Rahmen der Strategie für die Gleichstellung von Frauen und Männern

- ein Follow-up zur Europäischen Strategie für Menschen mit Behinderungen, die vor allem auf die Beseitigung von Barrieren abzielt

- die Fortsetzung von Aktivitäten im Bereich der Wohnungslosigkeit.

Der Katalog der von der Kommission in diesem Zusammenhang genannten Instrumente zeigt, dass die Plattform auf nicht rechtsverbindliche Instrumente setzt.

Die Plattform soll schließlich die politischen Impulse nutzen, die vom Europäischen Jahr zur Bekämpfung von Armut und sozialer Ausgrenzung 2010 ausgegangen sind. Vor diesem Hintergrund ist es konsequent, dass sehr stark auf die Einbeziehung der "Sozialen OMK « in die Strategie "Europa 2020 « abgestellt wird, welche die anderen Instrumente sozialpolitischer Intervention sowie die Zusammenarbeit zwischen den Mitgliedstaaten auf Regierungsebene ergänzt. Dabei zielt die OMK darauf ab, die Zusammenarbeit zwischen den Mitgliedstaaten durch den Austausch von Erfahrungen über "gute « und »beste « Praktiken zu verstärken und die Mitgliedstaaten zu veranlassen, sich freiwillig auf gemeinsame Zielsetzungen, Grundsätze und Vorgaben zu einigen, die in nationale Aktionspläne münden.

Wie im Falle der Europäischen Beschäftigungspolitik, die im AEU-Vertrag rechtlich verankert ist (20), ist die OMK Soziale Inklusion - Bekämpfung von Armut und sozialer Ausschließung - wie die OMK Alterssicherung und die OMK Gesundheit und Langzeitpflege - eine bereichsspezifisch ausgestaltete Strategie, die u. a. auf einem »Moni- toring « des Fortschritts bei der Erreichung vereinbarter Ziele basiert und folgende Schritte umfasst:

- Festlegung von Leitlinien für die Union zur Erreichung kurz- und langfristiger Ziele unter Verwendung von Indikatoren, die Reformschritte widerzuspiegeln geeignet sind

- Entwicklung sowohl quantitativer als auch qualitativer Indikatoren sowie von Benchmarks, die einen Vergleich sowohl der Mitgliedstaaten untereinander als auch der Mitgliedstaaten mit Drittstaaten ermöglichen

- Umsetzung der Europäischen Leitlinien in nationale Politik durch die Formulierung von Zielen und den Erlass von Maßnahmen auf nationaler Ebene, wobei freilich den Unterschieden zwischen den Mitgliedstaaten - etwa im Hinblick auf das »historische Gewachsensein« der Sozialschutzsysteme einschließlich der Mindestsicherungssysteme, m.a.W. der sozialpolitischen »Pfadabhängigkeit «, sowie auch der wirtschaftlichen Leistungskraft, dem Niveau des Sozialleistungssystems u.a. Rechnung getragen werden muss

- regelmäßiges »Monitoring « und »Reviewing « im Rahmen eines wechselseitigen Prozesses des Hin- und Herschauens und des Austausches von Erfahrungen

- dabei Berücksichtigung der Kompetenzordnung im Hinblick auf die Zuständigkeiten zwischen EU und Mitgliedstaaten sowie Beachtung des Subsidiaritätsprinzips mit der Folge, dass die Primärzuständigkeit der Mitgliedstaaten für den Sozialschutz und damit auch für die soziale Mindestsicherung respektiert wird.

Dabei setzt die OMK soziale Inklusion vor allem auf die Heranführung der Klienten an und ihre Einbeziehung in das Erwerbsleben. (21)

\section{Soziale Gerechtigkeit - wo steht Deutschland?}

Der Prozess des Hin- und Herschauens und des wechselseitigen Lernens soll an einem konkreten Beispiel illustriert werden: Anfang 2011 ist eine Untersuchung vorgelegt worden, die sich mit der sozialen Gerechtigkeit in den OECD-Ländern beschäftigt und 
anhand von Indikatoren versucht hat, einen sogenannten Gerechtigkeitsindex zu ermitteln. (22) Für Deutschland wird diesbezüglich ein Nachholbedarf konstatiert, kommt die Bundesrepublik doch auf dem dort entwickelten Gerechtigkeitsindex von 31 OECD-Staaten lediglich auf den 15. Platz. "Spitzenreiter" sind die nordischen Staaten und die Niederlande; auch die Schweiz, Frankreich, Österreich, Großbritannien und Luxemburg rangieren vor Deutschland.

Auch wenn man derartige Ranglisten wegen der Schwierigkeiten methodischer Art, einen solchen Index zu erstellen und seine einzelnen Komponenten zu gewichten und zu messen, mit großer Vorsicht zur Kenntnis nehmen sollte, sind doch einige Feststellungen recht aufschlussreich und plausibel.

Die verwandten einzelnen Zieldimensionen sozialer Gerechtigkeit sind Armutsvermeidung, Bildungszugang, Arbeitsmarktinklusion, Soziale Kohäsion und Gleichheit sowie Generationengerechtigkeit.

Die Zunahme der Altersarmut in Deutschland in den vergangenen Jahren ist wohl unbestritten, wie einige andere Untersuchungen zeigen. Entsprechendes gilt für die Kinderarmut, wo Deutschland in Europa einen - wenn nicht sogar den - Spitzenplatz einnimmt. Hier besteht offenkundiger Handlungsbedarf, da Einkommensarmut zwar nicht alle Dimensionen von Armut abdeckt, aber insofern eine wichtige Dimension ist, als ohne hinreichendes Einkommen soziale Teilhabe nicht möglich ist. Auch im Hinblick auf den Zugang zur Bildung gibt es nach wie vor Defizite, wie nicht zuletzt die PISA-Studien schon vor Jahren belegt haben.

Hingegen ist die Inklusion in den Arbeitsmarkt, insbesondere was Jugendliche angeht, hierzulande in höherem Maße gewährleistet als in anderen Mitgliedstaaten, wenn es auch für Geringqualifizierte und Langzeitarbeitslose nach wie vor schwer ist, auf dem Arbeitsmarkt Fuß zu fassen.

Im Hinblick auf soziale Kohäsion und Gleichheit fällt die Ungleichverteilung der Einkommen auf, die in den letzten Jahren zugenommen hat und den sozialen Zusammenhalt infrage stellt. (Darauf hat jüngst unter dem Blickwinkel des Steuerrechts auch der so apostrophierte »Professor aus Heidelberg« hingewiesen, der ja bisher

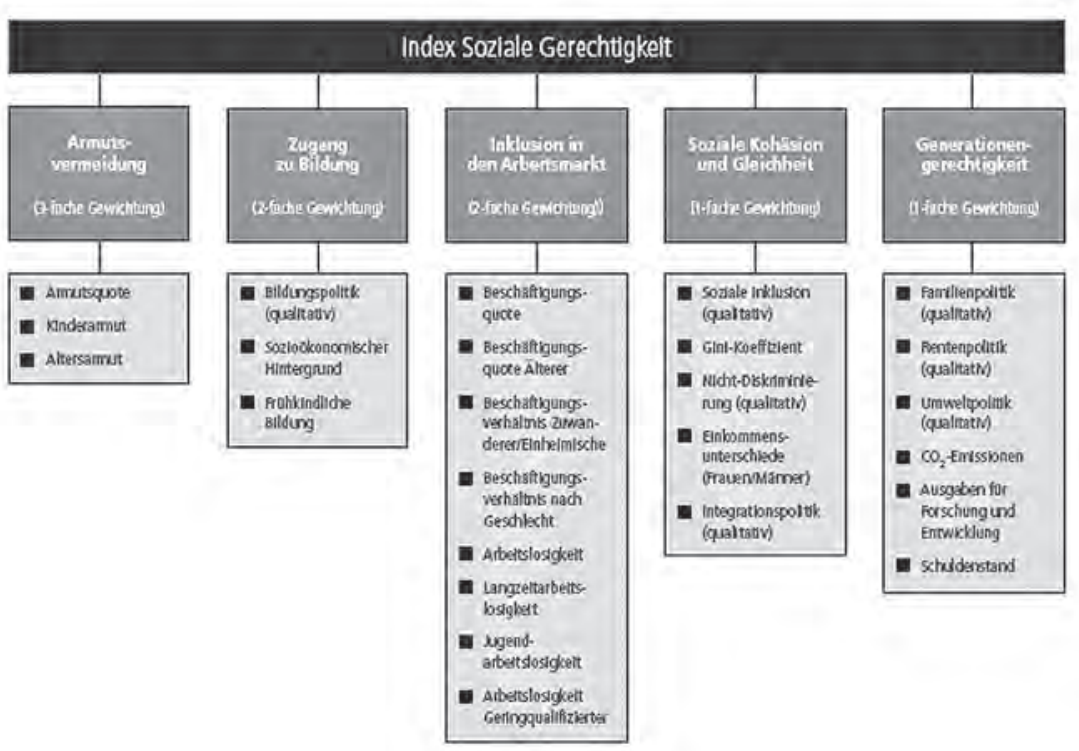

Aus 150 Kriterien setzt sich das Indikatorenset zusammen, mit dem die BertelsmannStiftung im vorigen Jahr die OECD-Länder in einem »Gerechtigkeitsindex« verglichen hat. Das ernüchternde Ergebnis: Von 31 Staaten landet die Wirtschaftsmacht Deutschland lediglich im Mittelfeld. Die 46-seitige Studie steht im Internet zum Herunterladen zur Verfügung.

www.bertelsmann-stiftung.de (Suchbegriff: Sustainable Governance Indicators)

nicht unbedingt als Vorkämpfer der sozialen Gerechtigkeit hervorgetreten ist. Anm. 22)

Demgegenüber scheint die Generationengerechtigkeit im internationalen Vergleich in vergleichsweise hohem Maße verwirklicht, ist doch die Staatsverschuldung und die damit einhergehende Belastung der nachfolgenden Generationen in finanzieller Hinsicht in Deutschland geringer als in den meisten anderen Mitgliedstaaten.

Die Verfasser der Studie weisen selber darauf hin, dass auch ein 150 Kriterien enthaltener Indikatorenset, wie er dieser Untersuchung zugrunde liegt, nicht den Anspruch erheben kann, die soziale Wirklichkeit zur Gänze widerzuspiegeln. Gleichwohl lassen sich mit der gewohnten Vorsicht gewisse Schlussfolgerungen darüber treffen, in welchen Bereichen besonderer Handlungsbedarf besteht, und es bietet sich an, in die OMK sozialer Schutz und soziale Eingliederung diese Befunde einfließen zu lassen.

Die Gewährleistung von sozialer Gerechtigkeit überhaupt, wie auch die Bekämpfung von Armut und sozialer Ausgrenzung im Besonderen, beziehen sich herkömmlicherweise auf den Nationalstaat als maßgeblichen Akteur. Materielle Chancengleichheit war insofern in der Vergangenheit ein partikulares Recht, als sie immer nur den Bürgern des jeweils eigenen Staates vorbehalten war, Ausländer hingegen von ihr i. d. R. ausgeschlossen blieben.

Die gegenwärtig unter dem Vorzeichen der Globalisierung vieldiskutierte Frage, ob die Idee der Gerechtigkeit für ihre Verwirklichung auf Souveränität und damit auf den Nationalstaat angewiesen ist oder aber ob es die Möglichkeit der Realisierung globaler sozialer Gerechtigkeit gibt (24), lässt sich m.E. in Bezug auf die »regionale Globalisierung « in Gestalt der Europäischen Integration im rechtlichen Rahmen der Europäischen Union dahingehend beantworten, dass es konkrete Ansätze $\mathrm{zu}$ »kontinentaler«, also europäischer Solidarität und sozialer Gerechtigkeit gibt und dass auch entsprechende Politiken bis hin zur Bekämpfung von Armut und sozialer Ausgrenzung nicht nur konzipiert, sondern auch praktiziert werden. (25)

Nicht zuletzt vor dem Hintergrund der aktuellen Entwicklung, namentlich der Euro-Krise, in der eine Option für die Fortentwicklung der Europäischen Union in einer Stärkung der Integration in wirtschaftlicher, zumal währungspolitischer und politischer Integration besteht, ist darüber nachzudenken, 
ob nicht auch zur Verhinderung eines weiteren Auseinanderklaffens von wirtschaftlicher und sozialer Integration an eine Stärkung der Legislativbefugnisse der Union nachgedacht werden muss.

Eine sozialpolitische Option bestünde dann darin, für ein Mehr an wirtschaftlichem, sozialem und territorialem Zusammenhalt zu sorgen. Denn es ist offensichtlich, "Zeit, europäisch zu denken ", sind doch die EU-Mitgliedstaaten in ihrer Souveränität bedroht und richtet sich die Hoffnung auf eine Stabilität von Geld und Recht auf die Europäische Union (26) - eine Erkenntnis, die, wenn sie denn richtig ist, auch nicht ohne Auswirkungen auf die Europäische Sozialpolitik bleiben kann.

\section{Anmerkungen}

(1) Der Artikel ist die Schriftfassung eines Referats, das der Verfasser auf der Fachtagung des Deutschen Vereins für öffentliche und private Fürsorge zum Thema »Aktuelle Entwicklungen der europäischen Sozialpolitik« im September 2011 gehalten hat.

(2) Art. 51 EWGV sowie Verordnungen (EWG) Nr. 3 und Nr. 4; später VOen (EWG) 1488/71 und 574/72 und heute die VOen 883/04 und 987/09.

(3) Art. 119 EWGV, heute Art. 157 AEUV.

(4) Art. 117 ff. EWGV; heute Art. 151 ff. AEUV.

(5) ABl. EG Nr. C 13 S. 1.

(6) Kommission der Europäischen Gemeinschaften, Schlussbericht von der Kommission an den Rat über das erste Programm von Modellvorhaben und Modellstudien zur Bekämpfung der Armut, Brüssel 1981.

(7) Vgl. dazu Hauser, R., Probleme und Ansatzpunkte einer gemeinsamen Politik zur Bekämpfung der Armut in der Europäischen Gemeinschaft, in: Borchardt, K./ Holzheu, F. (Hg.), Theorie und Politik der internationalen Wirtschaftsbeziehungen. Hans Möller zum 65. Geburtstag, Stuttgart 1980, S. 229 ff.; ders., Möglichkeiten und Probleme der Sicherung eines Mindesteinkommens in den Mitgliedstaaten der Europäischen Gemeinschaft, Frankfurt am Main
1983; aus juristischer Sicht dazu Schulte, B., Sozialpolitik in der Europäischen Gemeinschaft, in: Gegenwartskunde 1983, SH 4, S. 81 ff.; ders., Politik der Armut - Internationale Perspektiven, in: Leibfried, S./Tenstedt, F. (Hg.), Politik der Armut und Die Spaltung des Sozialstaats, Frankfurt am Main 1985, S. 383 ff.; ders., Europäische Sozialpolitik - eine Zwischenbilanz, in: Sozialer Fortschritt (SF) 1986.

(8) Vgl. dazu Hauser, R./CremerSchäfer, H./Nouvertné, U., Armut, Niedrigeinkommen und Unterversorgung in der Bundesrepublik Deutschland. Bestandsaufnahme und sozialpolitische Perspektiven (Studie erstellt im Auftrag der Kommission der Europäischen Gemeinschaften), Frankfurt am Main 1981; auch Hauser, R. Grundprobleme und Grundansatzpunkte der Armutsbekämpfung in der Bundesrepublik Deutschland, in: Armut in der Bundesrepublik Deutschland. Loccumer Protokolle 25 (1982), S. 3 ff.; ders., Armut im Wohlfahrtsstaat - empirischer Befund und Lösungsansätze, in: Lampert, H./Kühlewind, G. (Hg.), Das Sozialsystem der Bundesrepublik Deutschland, Nürnberg 1984, S. $214 \mathrm{ff}$

(9) Brüssel 1989.

(10) ABl. EG 1992 Nr. L 92/441.

(11) ABl. EG 1992 Nr. L 92/446.

(12) Vgl. dazu im Einzelnen jüngst Schulte, B., Soziale Mindestsicherung in den Mitgliedstaaten der Europäischen Union, in: Pfeil, W./Wöss, J. (Hg.), Bedarfsorientierte Mindestsicherung. Gesetze und Kommentare, Wien 2011, S. $225 \mathrm{ff}$.

(13) Art. 3 Abs. 5 VO 883/04.

(14) Vgl. Art. 7 Abs. 2 VO 1612/68.

(15) Vgl. Art. 2 VO 883/04.

(16) Vgl. dazu jüngst Peyrl, J., Europarechtliche und fremdenrechtliche Fragen der Mindestsicherung, in: Pfeil, W./Wöss, J. (Hg.), Handbuch Bedarfsorientierte Mindestsicherung, Wien 2011, S. $171 \mathrm{ff}$.

(17) Vgl. dazu jüngst Schulte, B., Sozialstaat Europa? Wege zu einer sozialen Werte- und Rechtsgemeinschaft in der Europäischen Union, in: Haerendel, U. (Hg.), Gerechtig- keit im Sozialstaat. Analysen und Vorschläge, Baden-Baden 2012.

(18) Vgl. dazu und zum Folgenden Europäische Kommission, Mitteilung »Europäische Plattform gegen Armut und soziale Ausgrenzung: Ein europäischer Rahmen für den sozialen und territorialen Zusammenhalt «, Brüssel, den 16.12.2010 (KOM 2010) 758 endg.

(19) Vgl. Europäische Kommission, Empfehlung zur aktiven Eingliederung der aus dem Arbeitsmarkt ausgegrenzten Personen (2008/867/EG), einschließlich gemeinsamer, vom Rat unterstützter Grundsätze für die aktive Eingliederung zugunsten einer wirksamen Armutsbekämpfung, Brüssel, den 17.12.2008.

(20) Vgl. Titel IX Beschäftigung (Art. 145-150 AEUV).

(21) Vgl. dazu etwa Wentzel, J./Wunder, A., Kohäsion oder Inklusion? $\mathrm{Zu}$ den Ansätzen der EU und des Europarates zur Bekämpfung von Armut und sozialer Ausgrenzung, in: Zeitschrift für europäisches Sozial- und Arbeitsrecht (ZESAR) 2009, S. 329 ff.

(22) BertelsmannStiftung (Hg.), Soziale Gerechtigkeit in der OECD - wo steht Deutschland? Sustainable Governance Indicators 2011, Gütersloh 2011.

(23) Vgl. Paul Kirchhof in der Frankfurter Allgemeinen Zeitung (FAZ) Nr. 196 v. 24.8.2011.

(24) Vgl. zu der Diskussion etwa Rasmussen, D., Die Möglichkeit globaler Gerechtigkeit, in: Forst, R./ Hartmann, M./Jaeggi, R./Saar, M. (Hg.), Sozialphilosophie und Kritik, Frankfurt am Main. 2009 m.w.N.

(25) Vgl. dazu auch Schulte a.a.O. (siehe Fn. 19).

(26) Vgl. in diesem Sinne Paul Kirchhof, Zeit, europäisch zu denken, in: FAZ Nr. 197 v. 25.8.2011. 